\title{
Multiobjective optimization and decision making in engineering sciences
}

\author{
Jussi Hakanen ${ }^{1} \cdot$ Richard Allmendinger $^{2}$
}

Received: 30 March 2021 / Revised: 31 March 2021 / Accepted: 31 March 2021 / Published online: 11 April 2021 (C) The Author(s) 2021

\begin{abstract}
Real-world decision making problems in various fields including engineering sciences are becoming ever more challenging to address. The consideration of various competing criteria related to, for example, business, technical, workforce, safety and environmental aspects increases the complexity of decision making and leads to problems that feature multiple competing criteria. A key challenge in such problems is the identification of the most preferred trade-off solution(s) with respect to the competing criteria. Therefore, the effective combination of data, skills, and advanced engineering and management technologies is becoming a key asset to a company urging the need to rethink how to tackle modern decision making problems. This special issue focuses on the intersection between engineering, multiple criteria decision making, multiobjective optimization, and data science. The development of new models and algorithmic methods to solve such problems is in the focus as much as the application of these concepts to real problems. This special issue was motivated by the 25th International Conference on Multiple Criteria Decision Making (MCDM2019) held in Istanbul, Turkey, in 2019.
\end{abstract}

Keywords Multiple criteria decision making - Evolutionary multiobjective optimization · Expensive optimization · Engineering sciences · Data science

Jussi Hakanen

jussi.hakanen@jyu.fi

Richard Allmendinger

richard.allmendinger@manchester.ac.uk

1 Faculty of Information Technology, University of Jyvaskyla, 40014 Jyvaskyla, Finland

2 The University of Manchester, Alliance Manchester Business School, Manchester M15 6PB, UK 


\section{Introduction}

Multiobjective optimization (MO) deals with optimization problems that have at least two (conflicting) objective functions to be optimized simultaneously. The conflict between the objectives means that their optimal per objective values cannot be obtained by a single solution and, thus, some balancing (trading off) between the objectives is needed. Mathematically, an MO problem can be formulated as:

$$
\begin{aligned}
& \min \mathbf{f}(\mathbf{x})=\left\{f_{1}(\mathbf{x}), \ldots, f_{k}(\mathbf{x})\right\} \\
& \text { s.t. } \mathbf{x} \in S,
\end{aligned}
$$

where the objective vector $\mathbf{f}$ consists of $k(\geq 2)$ objective functions $f_{i}: \mathbb{R}^{n} \rightarrow \mathbb{R}, i=1, \ldots, k$. The vector $\mathbf{x}=\left(x_{1}, \ldots, x_{n}\right)^{T}$, consisting of $n$ decision variables, is constrained by the feasible region $S \subset \mathbb{R}^{n}$.

The optimal solutions for problem (1) are often defined by using Pareto optimality. A feasible solution $\mathbf{x}^{*} \in S$ is called Pareto optimal if there does not exist another feasible solution $\mathbf{x} \in S\left(\mathbf{x} \neq \mathbf{x}^{*}\right)$ such that $f_{i}(\mathbf{x}) \leq f_{i}\left(\mathbf{x}^{*}\right)$ for all $i=1, \ldots, k$ and $f_{j}(\mathbf{x})<f_{j}\left(\mathbf{x}^{*}\right)$ for at least one $j$. The set of all Pareto optimal solutions is said to form the Pareto set, whose image in the objective space is known as the Pareto front. All Pareto optimal solutions are mathematically incomparable without any additional information.

When solving real-world MO problems, the idea is often to find a Pareto optimal solution or a small number of Pareto optimal solutions that are most preferred in the context of the problem considered. Then, a decision maker (DM) is a person who has the knowledge and understanding of the problem domain and can provide preference information regarding the conflicting objectives. There exist many methods for solving MO problems and they can be divided into categories based on the role of the DM in helping the methods to find the most preferred solution (Miettinen 1999): (1) In a priori methods, the DM is first asked to provide preferences that are then taken account in optimization to find a Pareto optimal solution that is closest to the preferences. (2) In a posteriori methods, an approximation of the whole Pareto front is first obtained and shown to the DM, who then selects the most preferred solution amongst them. (3) In interactive methods, the DM provides preference information iteratively and thus provides feedback to the method during the optimization (Miettinen et al. 2016).

Both mathematical and evolutionary optimization approaches have been used to solve MO problems. Mathematical optimization methods (Miettinen 1999) are efficient when functions are differentiable and gradient information is available or the structure of the problem can be exploited, while evolutionary optimization methods (Deb 2005; Emmerich and Deutz 2018) perform well on problems where not much or no information of the problem structure is available (e.g. black-box problems) or the problem features non-standard properties such as noisy/uncertain functions and multiple objectives (as they allow for the discovery of multiple Pareto optimal solutions in a single algorithmic run). Further, mathematical optimization methods offer guarantees of converge, especially for convex problems, while evolutionary optimization methods do not guarantee convergence but are well suited for non-convex 
problems and can provide a set of solutions for a single optimization run. Good results have been obtained by hybridizing these two approaches (Branke et al. 2008).

When solving real-world MO problems, a common challenge is the non-availability of closed-form expressions of the objective and/or constraint functions, also known as black-box functions. Consequently, the evaluation of these functions may involve the execution of computer simulations and/or real (physical, biological, chemical) experiments. Such evaluations are typically time-consuming, costly and/ or resource-intense, forcing the optimizer to arrive at a good solution within a limited number of evaluations. Hence, a lot of thought goes into deciding which solutions to evaluate at each iteration of the optimization. A common approach to cope with expensive objective/constraint function(s) is to replace them with a cheap to evaluate surrogate model (Allmendinger et al. 2017; Chugh et al. 2019). By optimizing over the surrogate model using an acquisition function, such as expected improvement, one can identify one or multiple solutions that are likely to lead to an improvement of the current best solution; these promising solutions can then be evaluated on the original expensive objective function.

In the presence of multiple objective functions, there is no reason to believe that the objectives share the same properties. In fact, the objectives may be heterogeneous varying in aspects such as evaluation time (slow vs fast to evaluate objectives) (Allmendinger et al. 2015), the induced landscape (rugged vs smooth), safety risks (causing serious consequences when evaluated below some safety threshold vs no consequences) (Kim et al. 2021), level of noise (deterministic vs noisy) and there may also be varying levels of correlations between the objectives throughout the search space; for a review of different types of heterogeneity please refer to Allmendinger and Knowles (2021). The use of surrogate models serves as a good starting point to cope with heterogeneous objectives. Surrogate models can also be used when the problem formulation has to be based only on some existing data (e.g. measurement data from experiments or sensors, business data, health data), and obtaining new data is either impossible or out of the hands of the optimizer; this scenario is also referred to as offline data-driven optimization as opposed to online data-driven optimization where the optimizer can decide when to evaluate which solution (Jin et al. 2019).

\section{Overview of the special issue articles}

The presence of multiple objectives in a problem increases the complexity of the decision making process as it requires both the optimizer and DM to trade-off multiple conflicting factors. An increase in the number of objectives poses also an additional layer of complexity when it comes to theoretical research in this domain. The eight papers in this special issue address both experimental and theoretical challenges arising in engineering sciences.

In Korondi et al. (2021) propose a novel optimization method for MO (and single-objective) problems featuring both expensive and uncertain evaluations of candidate solutions. The problems under investigation have highly limited computational budgets. A multi-fidelity surrogate-assisted approach is proposed, and has shown, 
on various test problems, to outperform standard surrogate techniques if cheap lowfidelity evaluations are available. The approach can be applied to problems where new data can be obtained by evaluating solutions, or where new data cannot be obtained but there is a surrogate model available of the problem under investigation. These are common setups in engineering applications.

An expensive MO problem was also considered by Prinz et al. (2021). More precisely, the authors consider the optimization of a mixing process in electrically conducting fluids. To tackle this problem, a trust-region method was modified to tackle a problem with multiple expensive objective functions. Moreover, the proposed method guarantees an improvement of the objective function values from a starting guess. The study allowed the authors not only to optimize the mixing process but also gain insight into the relationship between the decision variables and the objectives.

A new approach to solve multi-attribute decision making problems with subjective preferences and non-preferences of the DM is proposed by Mehlawat et al. (2021). Triangular fuzzy preference relations and triangular fuzzy non-preference relations are used to represent the subjective preferences and non-preferences, respectively. The proposed approach is validated using a multi-attribute decision making problem from the literature, and compared against similar existing approaches with good results being reported.

In Sekizaki et al. (2021) propose a novel methodology to tackle a many-objective engineering problem in the area of network reconfiguration of an electric distribution system. The challenge in the application considered is to determine how best to downsize equipment (distribution lines) such that costs are reduced while feasibility of the network reconfigurations (due to maintenance and/or replacement of distribution lines) is guaranteed. The proposed methodology utilises a modified version of the NSGA-III algorithm (Deb and Jain 2014) to identify a set of trade-off solutions to be presented to a DM (distribution system operator in this case), who then makes a final decision on which distribution lines to downsize. In collaboration with a power grid company, the authors then validate the methodology on a large-scale distribution system model and identify, within reasonable computational time, distribution lines suitable for downsizing. This very practical approach can assist the distribution system operator in long-term planning.

Yet another real-world application is addressed in the manuscript of Ortiz-Martin et al. (2021). Here the application is concerned with the design of a wastewater treatment process such that two objectives, operational costs and effluent quality, are optimized. The authors formulate this problem as a dynamic MO problem with the aim being to find the time varying control profiles (aeration factor in the last aerated reactor and the internal recycle flow rate) in order to optimize the objectives. An NSGA-II algorithm (Deb et al. 2002) coupled with scenario-based simulations is used to solve the problem. This methodology is of particular use in situations where the energy costs are fluctuating hourly, supplying the decision maker a set of possible wastewater treatment processes to implement depending on the actual economical, technical or legal circumstances.

The design of a fin to optimize its effectiveness and efficiency using MO is considered by Weerasena et al. (2021). The authors use a piecewise linear 
approximation of the design of a fin. The problem itself is then optimized using $\epsilon$ -constraint method. It is demonstrated that the approach is able to find trade-off solutions of similar quality regardless of the initial design of the fin and the Biot number (a dimensionless index used in heat transfer calculations). This research supports the design of optimized fins and, as noted by the authors, the discretization method proposed can also find application in other areas of heat transfer and mechanics.

In Yano and Nishizaki (2021) focus on the application of MO concepts to twolevel programming problems, also known as Stackelberg games. In these games, a leader (e.g. natural gas shipping company) chooses a strategy to start off, subsequently prompting a follower (e.g. pipeline operating company) to choose a strategy having knowledge of the leader's decision. The authors augment such problems with the notion of simple resources, which are shortages and excesses arising from the violation of constraints with discrete random variables. The simple resources are accounted for in the objective functions as penalty functions. By combining the concept of Pareto Stackelberg solutions with the Kuhn-Tucker approach, the authors are able to formulate the problem as a mixed-integer programming problem, which they solve using an interactive algorithm. Numerical experiments show that the proposed methodology can react efficiently to simple resource changes.

Finally, in Schultes et al. (2021) tackle a bi-objective shape optimization problem. The goal is to maximize the mechanical stability of a ceramic component under tensile load while minimizing its volume. Hypervolume scalarization is suggested to tackle the (discretized) optimization problem as it does not require an explicit handling of additional constraints and, compared to the weighted sum approach, allows for the discovery of unsupported non-dominated solutions. When augmenting hypervolume maximization on an iterative ascent algorithm, the authors show that for convex problems a non-trivial reference set generating the set of properly non-dominated solutions can be determined without prior knowledge on the Pareto front. The approach is also validated on a real ceramic component design problem allowing the authors to gain valuable insights into the relationship between the component shape and its reliability and cost.

\section{Conclusions}

The articles in the special issue cover a wide range of topics related to $\mathrm{MO}$, including both mathematical and evolutionary optimization approaches, preference elicitation techniques, approaches for solving expensive optimization problems, and approaches drawing from game theory. A strong engineering aspect is also present since many of the papers are dealing with a concrete real-world case study, which emphasizes a clear need for MO approaches in engineering sciences. It is also great to see that in many of the papers, the decision making aspect was present since the authors were not just happy to only produce Pareto optimal solutions but did also consider the decision making aspect of identifying practically relevant solutions. To summarize, MO has become an essential tool in many fields not least in engineering sciences, and it will continue to provide a platform to rely on in the future. 
This special issue was motivated by the MCDM2019 conference held in Istanbul, Turkey, which belongs to the trademark bi-annual conference series of the International Society on Multiple Criteria Decision Making. We highly appreciate the support from the conference organizers. Altogether, 27 manuscripts were submitted for the special issue of which 8 were finally accepted. This gives an acceptance rate of about $30 \%$. We would like to thank all the reviewers that were spending their precious time and provided high-quality reviews helping us to identify the manuscripts that were accepted for this special issue.

Open Access This article is licensed under a Creative Commons Attribution 4.0 International License, which permits use, sharing, adaptation, distribution and reproduction in any medium or format, as long as you give appropriate credit to the original author(s) and the source, provide a link to the Creative Commons licence, and indicate if changes were made. The images or other third party material in this article are included in the article's Creative Commons licence, unless indicated otherwise in a credit line to the material. If material is not included in the article's Creative Commons licence and your intended use is not permitted by statutory regulation or exceeds the permitted use, you will need to obtain permission directly from the copyright holder. To view a copy of this licence, visit http://creativecommons.org/licen ses/by/4.0/.

\section{References}

Allmendinger R, Emmerich M, Hakanen J, Jin Y, Rigoni E (2017) Surrogate-assisted multicriteria optimization: complexities, prospective solutions, and business case. J Multi-Criteria Decis Anal 24(1-2):5-24

Allmendinger R, Handl J, Knowles J (2015) Multiobjective optimization: when objectives exhibit nonuniform latencies. Eur J Oper Res 243(2):497-513

Allmendinger R, Knowles J (2021) Heterogeneous objectives: state-of-the-art and future research. Arxiv preprint arXiv:2103.15546 [cs.NE]

Branke J, Deb K, Miettinen K, Slowiński R (eds) (2008) Multiobjective optimization: interactive and evolutionary approaches. Springer, Berlin

Chugh T, Sindhya K, Hakanen J, Miettinen K (2019) A survey on handling computationally expensive multiobjective optimization problems with evolutionary algorithms. Soft Comput 23(9):3137-3166

Deb K (2005) Multiobjective optimization using evolutionary algorithms. Wiley, New York

Deb K, Jain H (2014) An evolutionary many-objective optimization algorithm using reference-pointbased nondominated sorting approach, part i: solving problems with box constraints. IEEE Trans Evol Comput 18(4):577-601

Deb K, Pratap A, Agarwal S, Meyarivan T (2002) A fast and elitist multiobjective genetic algorithm: NSGA-II. IEEE Trans Evol Comput 6(2):182-197

Emmerich MTM, Deutz AH (2018) A tutorial on multiobjective optimization: fundamentals and evolutionary methods. Natural Comput 17:585-609

Jin Y, Wang H, Chugh T, Guo D, Miettinen K (2019) Data-driven evolutionary optimization: an overview and case studies. IEEE Trans Evol Comput 23:442-458

Kim Y, Allmendinger R, Lopez-Ibanez M (2021) Safe learning and optimization techniques: Towards a survey of the state of the art. Arxiv preprint arXiv:2101.09505 [cs.LG]

Korondi PZ, Marchi M, Poloni C, Parussini L (2021) Multi-fidelity design optimisation strategy under uncertainty with limited computational budget. Optimization and Engineering. https://doi.org/10. 1007/s11081-020-09510-1

Mehlawat MK, Gupta P, Ahemad F (2021) A nonlinear programming approach to solve MADM problem with triangular fuzzy preference and non-preference information. Optim Eng. https://doi.org/10. 1007/s11081-020-09524-9

Miettinen K (1999) Nonlinear multiobjective optimization. Kluwer, Dordrecht 
Miettinen K, Hakanen J, Podkopaev D (2016) Interactive nonlinear multiobjective optimization methods. In: Greco S, Ehrgott M, Figueira JR (eds) Multiple criteria decision analysis, 2nd edn. Springer, Berlin, pp 927-976

Ortiz-Martin VM, Frutos JM, Hontoria E, Hernandez-Fernandez FJ, Egea JA (2021) Multiplicity of solutions in model-based multiobjective optimization of wastewater treatment plants. Optim Eng. https://doi.org/10.1007/s11081-020-09500-3

Prinz S, Thomann J, Eichfelder G, Boeck T, Schumacher J (2021) Expensive multi-objective optimization of electromagnetic mixing in a liquid metal. Optim Eng. https://doi.org/10.1007/ s11081-020-09561-4

Schultes J, Stiglmayr M, Klamroth K, Hahn C (2021) Hypervolume scalarization for shape optimization to improve reliability and cost of ceramic components. Optim Eng. https://doi.org/10.1007/ s11081-020-09586-9

Sekizaki S, Yamasaki T, Nishizaki I, Hayashida T, Kanazawa Y, Hikoyama K, Uenishi H (2021) Distribution line downsizing based on a set of non-dominated solutions for a network reconfiguration problem of an electric distribution system with many objectives. Optim Eng. https://doi.org/10. 1007/s11081-020-09560-5

Weerasena L, Belinskiy BP, Hiestand JW (2021) Optimal effectiveness and efficiency of a fin in steadystate: multiobjective approach. Optim Eng. https://doi.org/10.1007/s11081-020-09497-9

Yano H, Nishizaki I (2021) Multiobjective two-level simple recourse programming problems with discrete random variables. Optim Eng. https://doi.org/10.1007/s11081-020-09532-9

Publisher's Note Springer Nature remains neutral with regard to jurisdictional claims in published maps and institutional affiliations. 